\title{
Adaptive Active Noise Suppression Using Multiple Model Switching Strategy
}

\author{
Quanzhen Huang, ${ }^{1}$ Suxia Chen, ${ }^{2}$ Mingming Huang, ${ }^{1}$ and Zhuangzhi Guo ${ }^{1}$ \\ ${ }^{1}$ School of Electrical Information Engineering, Henan Institute of Engineering, Zhengzhou 451191, China \\ ${ }^{2}$ School of Computer, Henan Institute of Engineering, Zhengzhou 451191, China \\ Correspondence should be addressed to Zhuangzhi Guo; guozhuangzhi2012@126.com
}

Received 21 September 2016; Revised 26 November 2016; Accepted 19 December 2016; Published 29 January 2017

Academic Editor: Jeong-Hoi Koo

Copyright ( 2017 Quanzhen Huang et al. This is an open access article distributed under the Creative Commons Attribution License, which permits unrestricted use, distribution, and reproduction in any medium, provided the original work is properly cited.

\begin{abstract}
Active noise suppression for applications where the system response varies with time is a difficult problem. The computation burden for the existing control algorithms with online identification is heavy and easy to cause control system instability. A new active noise control algorithm is proposed in this paper by employing multiple model switching strategy for secondary path varying. The computation is significantly reduced. Firstly, a noise control system modeling method is proposed for duct-like applications. Then a multiple model adaptive control algorithm is proposed with a new multiple model switching strategy based on filter- $\mathrm{u}$ least mean square (FULMS) algorithm. Finally, the proposed algorithm was implemented on Texas Instruments digital signal processor (DSP) TMS320F28335 and real time experiments were done to test the proposed algorithm and FULMS algorithm with online identification. Experimental verification tests show that the proposed algorithm is effective with good noise suppression performance.
\end{abstract}

\section{Introduction}

Nowadays noise cancellation becomes more and more important for industrial applications such as engines, blowers, fans, transformers, and compressors [1-6]. The traditional passive noise cancellation techniques using sound absorbent materials can suppress high-frequency acoustic noises effectively, usually higher than $500 \mathrm{~Hz}$ [7]. But, they are ineffective or tend to be bulky for low frequency cases. Meanwhile the passive noise control equipment is relatively large and expensive. In contrast with the passive noise suppression methods, active noise control techniques have excellent low frequency characteristic, with potential benefits in size, weight, and cost [8].

The idea of active noise control using a microphone and loudspeaker was first proposed in a 1936 patent by Lueg. In the 1980s, the development of solid state electronics and digital signal processing technologies enabled low-cost implementation of powerful adaptive algorithms and encouraged widespread development and application of active noise control systems. While the characteristics of the noise source and the acoustic environment are time varying, an active noise control system is required to track these changes and uncertainties. In particular, the secondary path between the loudspeakers and error sensors is time varying as the temperature and noise propagation condition would vary even though the noise source does not change. So in most actual applications, simultaneous secondary path identification should be done.

Various algorithms can be used for this purpose depending on the problem setting and the allowed computational complexity. The most famous one is Filtered-x Least Mean Squares (FxLMS) algorithms, which is proposed by different researchers independently. Several theoretical developments and successful applications are documented in the related literature [3,9-13]. FxLMS is used to adapt FIR filter coefficients of a controller; however the IIR adaptive filter is preferred in case the optimal controller has one or more poles close to the unit circle which may yield a very lone FIR optimal controller. In case the plant to be controlled contains intrinsic acoustical 


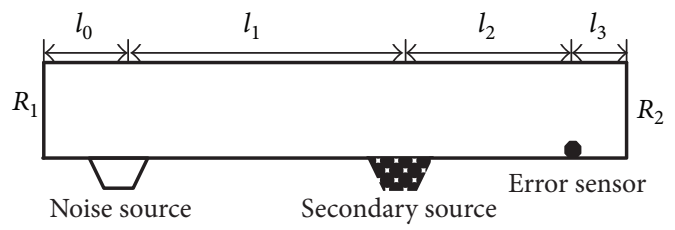

FIGURE 1: Schematic diagram for a duct-like application.

feedback especially and the optimal controller might contain unstable poles, it is even impossible to use an FIR adaptive filter to do optimal control without reducing the bandwidth of the controller. Many adaptive online identification methods are reported [14-18]. A functional-link-artificial-neuralnetwork- (FLANN-) based multichannel nonlinear active noise control (ANC) system trained using a particle swarm optimization (PSO) algorithm suitable for nonlinear noise processes is proposed in [19]. A novel online secondary path modeling scheme based on the Generalized Levinson Durbin (GLD) algorithm is proposed in [20]. A new adaptive strategy based on fractional signal processing is proposed using multidirectional step size fractional least mean square algorithm for online secondary path modeling in [21]. A simplified subband ANC algorithm without secondary path modeling is proposed in [22].

But most of them could not be applied to the cases while secondary path varies rapidly and not suited for multiple input multiple output applications with too many unknown parameters.

To solve these problems, a new adaptive multiple model switching control algorithm is proposed. The paper is organized as follows: Section 2 introduces the active noise control system modeling method for duct-like applications. Section 3 introduces the FULMS algorithm. Section 4 proposes a multiple model switching strategy. Section 5 gives the real time test results. Section 6 comes the conclusion.

\section{Active Noise Control System Modeling for Duct-Like Applications}

Schematic diagram of active noise control system for a ductlike application is shown in Figure 1. The sound incident from the left noise source is picked up by the microphone and after some processing by the active noise controller, this signal is fed to the secondary sources (loudspeaker) such that to the right side the primary signal and the additional signal cancel each other. The noise source is located at $l_{0}$ from one end of the duct, and an error sensor is located at $l_{3}$ from the other end of the duct. The secondary source is located at $l_{1}$ form the noise source. The pressure reflection coefficients in two ends are $R_{1}$ and $R_{2}$, respectively. The primary path between the noise source and the secondary source is $P$; the secondary path between the secondary source and error sensor is $S$.

According the steady state travelling wave theory, the frequency response for secondary path and the primary path can be obtained as follows:

$$
\begin{aligned}
& S(j w) \\
& \quad=\frac{H_{s} H_{e} e^{-k l_{2}}\left[1+D_{e} R_{2} e^{-2 k l_{3}}\right]\left[1+D_{s} R_{1} e^{-2 k\left(l_{0}+l_{1}\right)}\right]}{1-R_{1} R_{2} e^{-2 k l}}, \\
& P(j w) \\
& \quad=\frac{H_{e} H_{p} e^{-k\left(l_{1}+l_{2}\right)}\left[1-D_{e} R_{2} e^{-2 k l_{3}}\right]\left[1+D_{p} R_{1} e^{-2 k l_{0}}\right]}{1-R_{1} R_{2} e^{-2 k l}} .
\end{aligned}
$$

Here $H_{p}$ is the electroacoustic transfer function of the noise source, $H_{s}$ is the electroacoustic transfer function of the secondary source, and $H_{e}$ is the electroacoustic transfer function of the error sensor. $D_{p}$ is the directivity factor of the noise source, $D_{s}$ is the directivity factor of the secondary source, and $D_{e}$ is the directivity factor of the error source. $k=j \omega / c$, in which $\omega$ is angular frequency and $c$ is sound speed. For $z$-transform,

$$
\begin{aligned}
& S(z)=\frac{z^{-n_{2}}\left[1+R_{2} z^{-2 n_{3}}\right]\left[1+R_{1} z^{-2\left(n_{0}+n_{1}\right)}\right]}{1-R_{1} R_{2} z^{-N}}, \\
& P(z)=\frac{z^{-\left(n_{1}+n_{2}\right)}\left[1+R_{2} z^{-2 n_{3}}\right]\left[1+R_{1} z^{-2 n_{0}}\right]}{1-R_{1} R_{2} z^{-N}} .
\end{aligned}
$$

Here, $n_{i}$ is the integer near to $l_{i} f_{s} / c$; here $f_{s}$ is sampling frequency of the control system; $c$ is the sound speed. $N=$ $2\left(n_{0}+n_{1}+n_{2}+n_{3}\right)$; while the sound speed changes with time, the whole system becomes time varying.

Unfolding (2),

$$
\begin{aligned}
& S(z)=\frac{z^{-n_{2}}+R_{1} z^{-2\left(n_{0}+n_{1}+0.5 n_{2}\right)}+R_{2} z^{-2\left(0.5 n_{2}+n_{3}\right)}+R_{1} R_{2} z^{-2\left(n_{0}+n_{1}+0.5 n_{2}+n_{3}\right)}}{1-R_{1} R_{2} z^{-N}}, \\
& P(z)=\frac{z^{-\left(n_{1}+n_{2}\right)}+R_{1} z^{-2\left(n_{0}+0.5 n_{1}+0.5 n_{2}\right)}+R_{2} z^{-2\left(0.5 n_{1}+0.5 n_{2}+n_{3}\right)}+R_{1} R_{2} z^{-2\left(n_{0}+0.5 n_{1}+0.5 n_{2}+n_{3}\right)}}{1-R_{1} R_{2} z^{-N}} .
\end{aligned}
$$

\section{FULMS Algorithm}

A block diagram of an adaptive IIR ANC system using FULMS algorithm is illustrated in Figure 2. The output signal of the IIR filter $y(n)$ is computed as

$$
y(n)=\mathbf{a}^{T}(n) x(n)+\mathbf{b}^{T}(n) y(n-1) .
$$




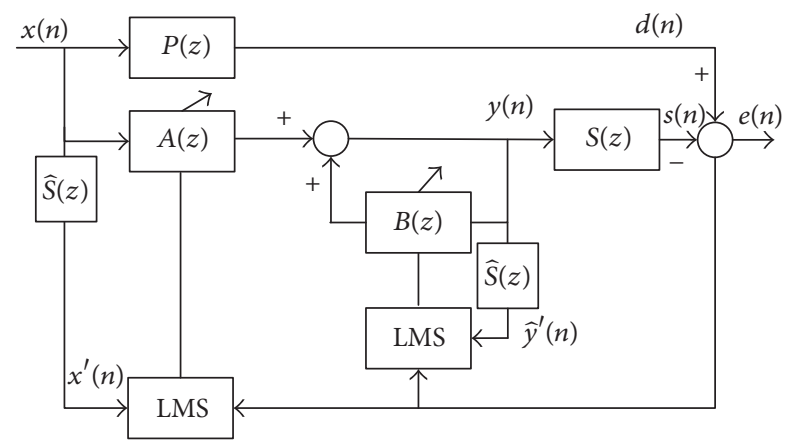

FIGURE 2: Schematic diagram for a duct-like application.

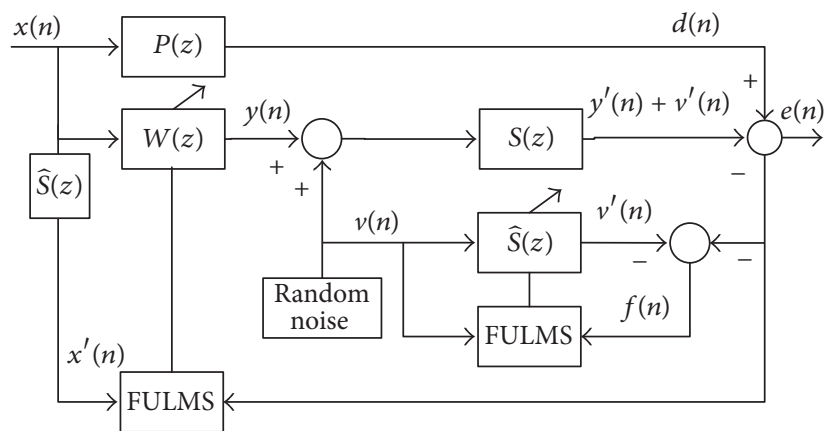

FIGURE 3: FULMS algorithm with online identification.

Here, $\mathbf{a}(n)=\left[\begin{array}{llll}a_{0}(n) & a_{1}(n) & \cdots & a_{L-1}(n)\end{array}\right]^{T}$ is the weight vector of $A(z)$ and $\mathbf{b}(n)=\left[\begin{array}{llll}b_{1}(n) & b_{2}(n) & \cdots & b_{M}(n)\end{array}\right]^{T}$ is the weight vector of $B(z)$. And FULMS algorithm can be adopted to find the optimal set of these coefficients:

$$
\begin{aligned}
& \mathbf{a}(n+1)=a(n)+\mu x^{\prime}(n) e(n), \\
& \mathbf{b}(n+1)=b(n)+\mu \widehat{y}^{\prime}(n-1) e(n) .
\end{aligned}
$$

Here $\widehat{y}^{\prime}(n-1)=\widehat{s}(n) * y(n-1)$.

Most of current researches are involved in online identification. A schematic diagram of FULMS algorithm with online identification is shown in Figure 3.

Zero-mean white noise $v(n)$ is used to drive the secondary source. Estimated $\widehat{S}(z)$ is used in parallel with the secondary path but only with a white noise input. The adaptive filters are updated using FULMS algorithm. After algorithm convergence, the residual noise will perturb the coefficients $\widehat{S}(z)$ and result in a misalignment:

$$
\sigma_{\widehat{s}}^{2} \equiv \lim _{n \rightarrow \infty} E \sum_{m=0}^{M-1}\left[\widehat{s}_{m}(n)-s_{m}(n)\right]^{2} \approx \frac{\mu}{2} M \sigma_{u}^{2} .
$$

Here, $\widehat{s}_{m}(n), s_{m}(n), m=0,1, \ldots, M-1$, are the impulse response of $\widehat{S}(z), S(z)$, respectively. Here $E$ means mathematical expectation. $\sigma$ means variance. $\mu$ is adaptive step size.

\section{Multiple Model Switching Strategy}

Multiple model adaptive control is a control strategy that can identify and adapt system changing characteristics. Multiple model strategy has an advantage over single model schemes because it selects the most suitable model among finite set of possible models. The computational burden does not much increase even though the number of channels increases. The block diagram of the proposed multiple model switching strategy is shown in Figure 4.

The residual error for $i$-th model is

$$
\varepsilon_{i}(n)=e(n)-s_{i}(n) .
$$

The power of the $i$-th error can be used to select the $\widehat{S}(z)$ :

$$
J_{i}(t)=\sum_{j=0}^{t} e^{-\lambda(t-j)} \varepsilon_{i}^{2}(j) .
$$

Here $\lambda$ is forgetting factor. $t$ is time index. Model bank contains a fixed model bank for estimation of $S(z)$. The identified model is fed to the block of $\widehat{S}(z)$, which is used to filter $x(n)$. LMS algorithm is employed to adjust the weights of filter A and filter B.

The model which has the minimum residual power is selected as current $\widehat{S}(z)$. The algorithm procedure can be shown as follows:

(1) Constructing a model bank with m fixed models

(2) Calculating $J_{i}$ for each model

(3) Selecting $\widehat{S}(z)$ which has the minimum residual power

(4) Employing the selected $\widehat{S}(z)$ into the FULMS algorithm to generate the filtered reference signals. The computation burden of the proposed multiple model strategy is significantly reduced as no adaptive model identification is carried out.

\section{Experimental Tests and Results}

To test the proposed algorithm, a test experimental platform is constructed using a 10-meter duct with two loudspeakers, two microphones, and an acoustic rate sensor. One loudspeaker is used to generate noise while canceling noise channeled through the other speaker. A microphone is placed in cancellation zone to pick up the resultant noise signal. The control paradigm is implemented on a Texas Instrument TMS320F28335 Digital Signal Processing chip. The experimental platform schematic diagram is shown in Figure 5. The sampling frequency is $10000 \mathrm{~Hz} . \mu=0.001$ and the length of adaptive filters is 24 .

At first, sound speed is measured for various conditions using the acoustic rate sensor, and minimum value is $325 \mathrm{~m} / \mathrm{sec}$ and the maximum value is $355 \mathrm{~m} / \mathrm{sec}$. Six fixed models are used to construct the model bank, and one model calculated using the current measured acoustic rate is used as the reference model to value the model switching performance. To accelerate the experiment, rather than waiting for condition change, an air conditioner is employed. 


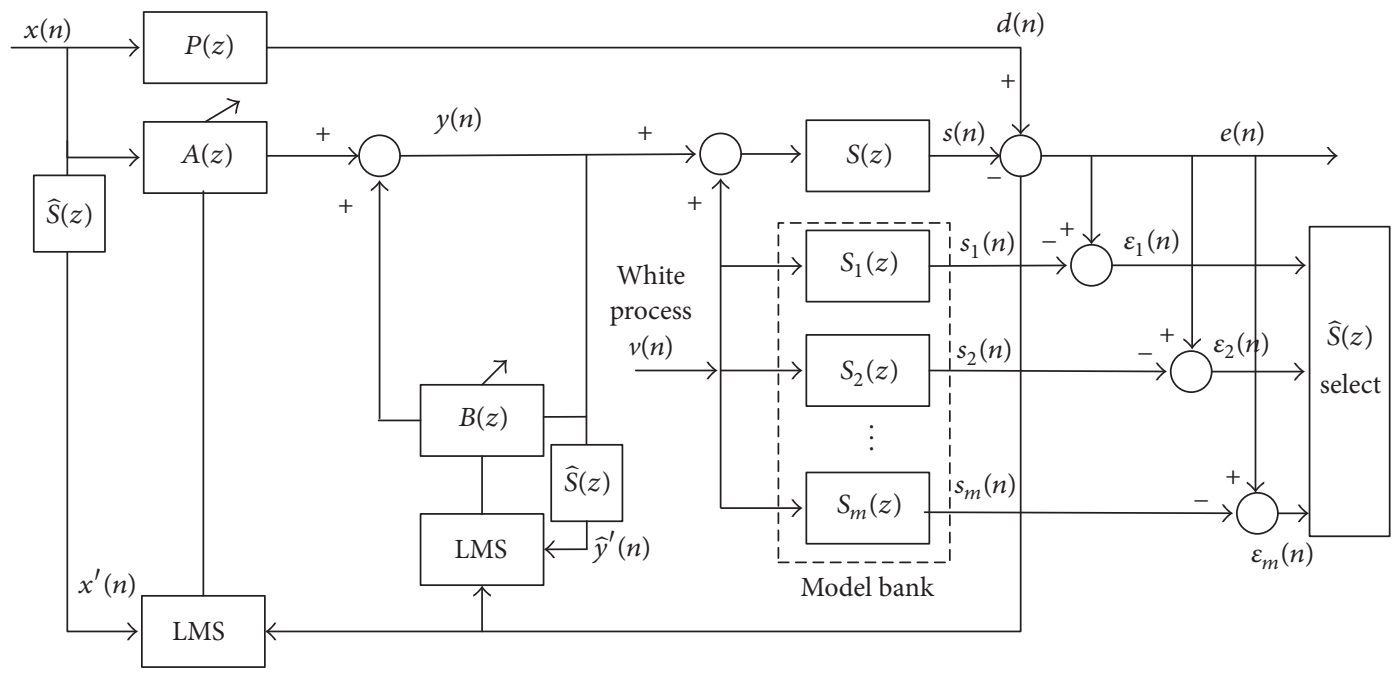

FIGURE 4: Block diagram of the proposed multiple model switching control system.

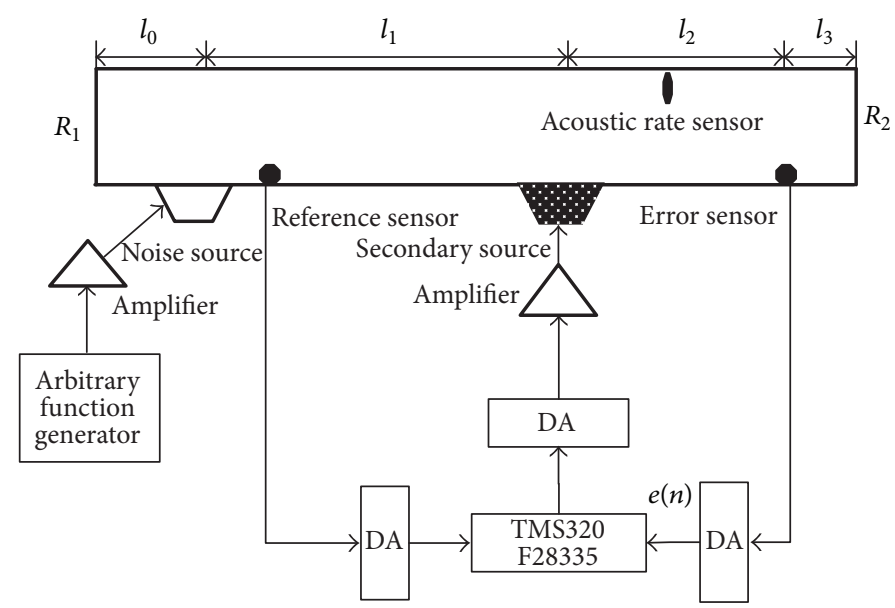

Figure 5: Experimental platform setup.

TABLE 1: Duct parameter value.

\begin{tabular}{lcc}
\hline Duct parameter & Symbol & Value \\
\hline Reflection coefficients & $R_{1}, R_{2}$ & $0.65 ; 0.65$ \\
Duct length & $l_{0}, l_{1}, l_{2}, l_{3}$ & $2.2 \mathrm{~m} ; 9.2 \mathrm{~m} ; 5.2 \mathrm{~m} ; 1.8 \mathrm{~m}$ \\
\hline
\end{tabular}

The duct parameters are shown in Table 1.

Two paradigms are tested on the experimental platform. The first one is the FULMS with online identification algorithm, while the model change rate is not so fast (the air conditioner changes slowly). The control performance is shown in Figure 6. It is clear that while the model change rate is slow, the control performance of FULMS algorithm with online identification is still acceptable. While the air conditioner changes fast, the control performance can be shown in Figure 7. The FULMS with online identification cannot assure the satisfactory control performance any more.

To test the performance of the proposed multiple model switching control algorithm, several experiments were done.

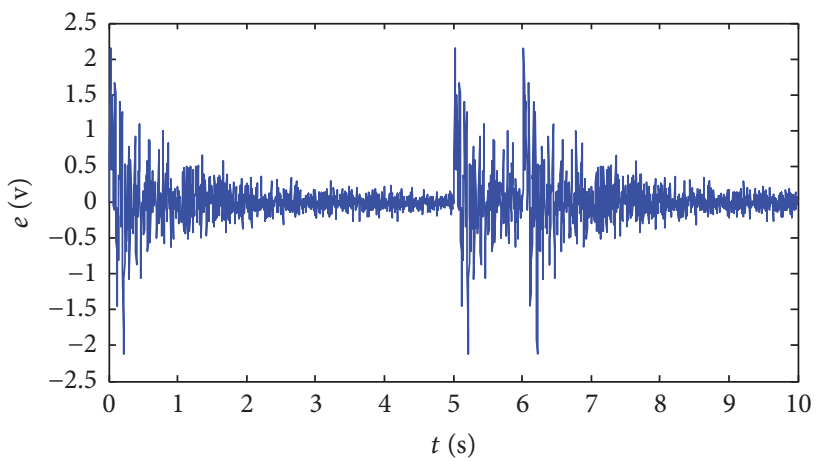

FIGURE 6: Control performance with slow model change.

The first experiment is designed to find a suitable memory length, three cases are listed in Figure 8, the memory length in Figure $8(a)$ is 100 , the memory length in Figure $8(b)$ is 1000 , and the memory length in Figure $8(c)$ is 5000 . The solid line is the model calculated using the current measured 


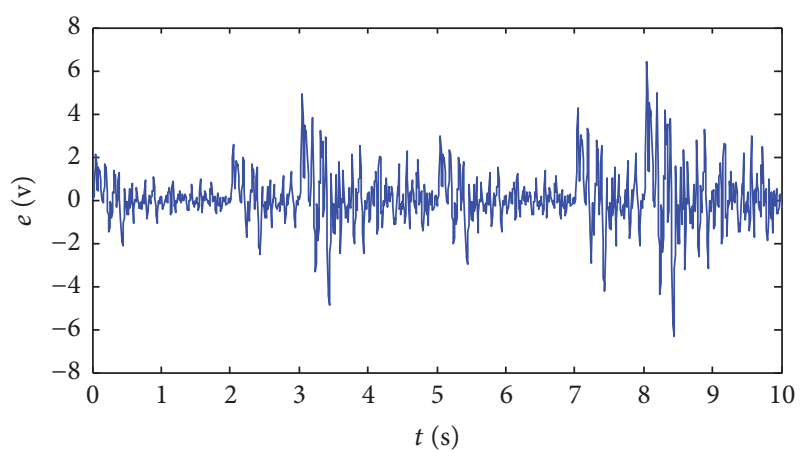

FIGURE 7: Control performance with fast model change.

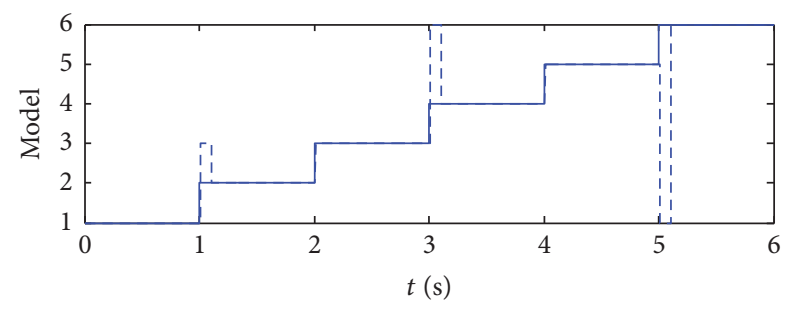

(a) Memory length is 100

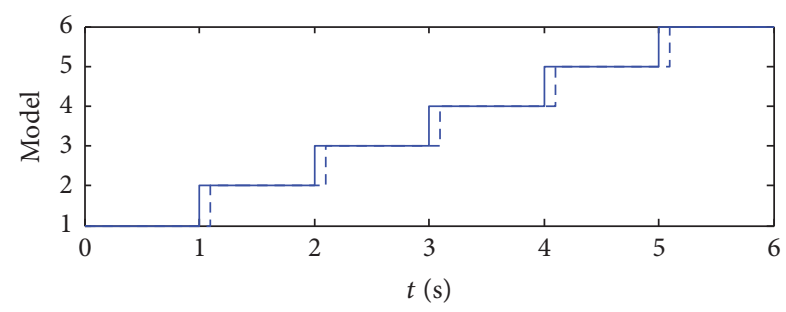

(b) Memory length is 1000

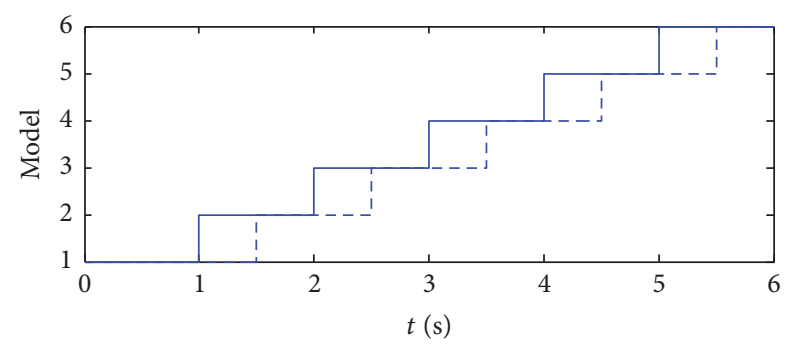

(c) Memory length is 5000

Figure 8: Model switching with different memory length.

acoustic rate, and it is rounded to the six fixed models. As shown in Figure 8, while the memory length is too short, the model switching is too fast and will cause instability. While the memory length is too long, the switching is too slow, and the model change rate is larger, it would also cause instability. The final control performance of our multiple model switching FULMS algorithm is shown in Figure 9. The proposed algorithm could guarantee a satisfactory suppression performance while the secondary path changes.

\section{Conclusion}

A novel active noise control algorithm is proposed in this paper by employing multiple model switching strategy for

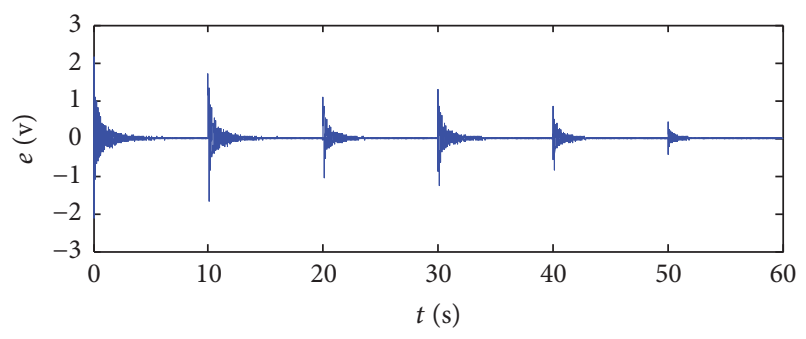

FIGURE 9: Control performance of the proposed method.

secondary path with noise control system modeling method for duct-like applications. Real time experiments were done. The experimental results show that the proposed algorithm has good noise suppression performance while the secondary path changes. The transient process of multiple model switching needs to be analyzed for nonlinear applications.

\section{Competing Interests}

The authors declare that they have no competing interests.

\section{Acknowledgments}

This research is supported by the National Nature Science Foundation of China (no. 61403123, no. 61305106), Technological Innovation Talents Projects of Henan Universities (no. 17HASTIT020), Henan Province Science and Technology Project (no. 162102210099, no. 162102310081), and Henan Province Youth Backbone Teachers Scheme in 2016 year.

\section{References}

[1] N. V. George and G. Panda, "Advances in active noise control: a survey, with emphasis on recent nonlinear techniques," Signal Processing, vol. 93, no. 2, pp. 363-377, 2013.

[2] Y. Li, X. Wang, and D. Zhang, "Control strategies for aircraft airframe noise reduction," Chinese Journal of Aeronautics, vol. 26, no. 2, pp. 249-260, 2013.

[3] Z. Bo, J. Yang, C. Sun, and S. Jiang, "A filtered-x weighted accumulated LMS algorithm: stochastic analysis and simulations for narrowband active noise control system," Signal Processing, vol. 104, pp. 296-310, 2014.

[4] I.-H. Yang, J.-E. Jeong, U.-C. Jeong, J.-S. Kim, and J.-E. Oh, "Improvement of noise reduction performance for a high-speed elevator using modified active noise control," Applied Acoustics, vol. 79, pp. 58-68, 2014.

[5] G. Nelson, R. Rajamani, and A. Erdman, "Noise control challenges for auscultation on medical evacuation helicopters," Applied Acoustics, vol. 80, pp. 68-78, 2014.

[6] Y. Kajikawa, W.-S. Gan, and S. M. Kuo, "Recent applications and challenges on active noise control," in Proceedings of the 8th International Symposium on Image and Signal Processing and Analysis (ISPA '13), Trieste, Italy, September 2013.

[7] S. Bianchi, A. Corsini, and A. G. Sheard, "A critical review of passive noise control techniques in industrial fans," Journal of Engineering for Gas Turbines and Power, vol. 136, no. 4, Article ID 044001, 2013. 
[8] D. Moreau, Active Control of Noise and Vibration, CRC Press, Second edition, 2012.

[9] G. Sun, T. Feng, M. Li, and T. C. Lim, "Convergence analysis of FxLMS-based active noise control for repetitive impulses," Applied Acoustics, vol. 89, pp. 178-187, 2015.

[10] B. Krstajic, Z. Zecevic, and Z. Uskokovic, "Increasing convergence speed of FxLMS algorithm in white noise environment," $A E U$ - International Journal of Electronics and Communications, vol. 67 , no. 10, pp. 848-853, 2013.

[11] T. Wang and W.-S. Gan, "Stochastic analysis of FXLMS-based internal model control feedback active noise control systems," Signal Processing, vol. 101, pp. 121-133, 2014.

[12] I. T. Ardekani and W. H. Abdulla, "Theoretical convergence analysis of FxLMS algorithm," Signal Processing, vol. 90, no. 12, pp. 3046-3055, 2010.

[13] I. T. Ardekani and W. H. Abdulla, "Filtered weight FxLMS adaptation algorithm: analysis, design and implementation," International Journal of Adaptive Control and Signal Processing, vol. 25, no. 11, pp. 1023-1037, 2011.

[14] P. Davari and H. Hassanpour, "Designing a new robust online secondary path modeling technique for feedforward active noise control systems," Signal Processing, vol. 89, no. 6, pp. 11951204, 2009.

[15] H. Hassanpour and P. Davari, "An efficient online secondary path estimation for feedback active noise control systems," Digital Signal Processing, vol. 19, no. 2, pp. 241-249, 2009.

[16] M. T. Akhtar, M. Abe, M. Kawamata, and A. Nishihara, "Online secondary path modeling in multichannel active noise control systems using variable step size," Signal Processing, vol. 88, no. 8, pp. 2019-2029, 2008.

[17] Q. Huang, J. Luo, Z. Gao, X. Zhu, and H. Li, "An improved filter$\mathrm{u}$ least mean square vibration control algorithm for aircraft framework," Review of Scientific Instruments, vol. 85, no. 9, Article ID 095003, 2014.

[18] G.-Y. Jin, T.-J. Yang, Y.-H. Xiao, and Z.-G. Liu, "A simultaneous equation method-based online secondary path modeling algorithm for active noise control," Journal of Sound and Vibration, vol. 303, no. 3-5, pp. 455-474, 2007.

[19] N. V. George and G. Panda, "A particle-swarm-optimizationbased decentralized nonlinear active noise control system," IEEE Transactions on Instrumentation and Measurement, vol. 61, no. 12, pp. 3378-3386, 2012.

[20] S. Tyagi, V. Katre, and N. V. George, "Online estimation of secondary path in active noise control systems using Generalized Levinson Durbin algorithm," in Proceedings of the 19th International Conference on Digital Signal Processing (DSP '14), pp. 552-555, IEEE, Hong Kong, August 2014.

[21] M. S. Aslam and M. A. Z. Raja, "A new adaptive strategy to improve online secondary path modeling in active noise control systems using fractional signal processing approach," Signal Processing, vol. 107, pp. 433-443, 2015.

[22] M. Gao, J. Lu, and X. Qiu, "A simplified subband ANC algorithm without secondary path modeling," IEEE/ACM Transactions on Audio, Speech, and Language Processing, vol. 24, no. 7, pp. 11641174, 2016. 


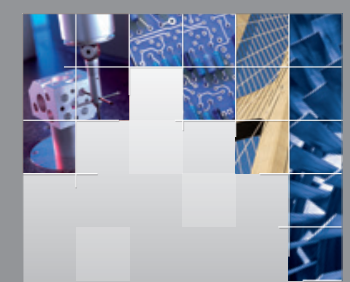

\section{Enfincering}
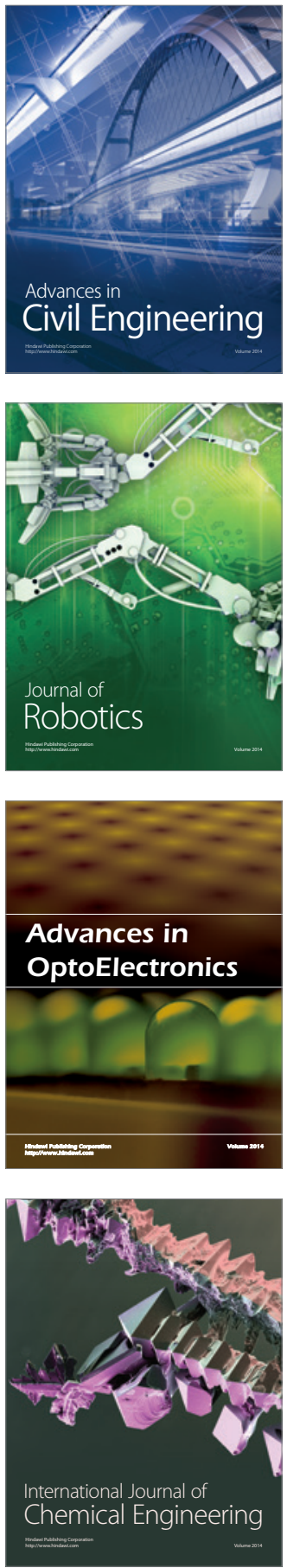

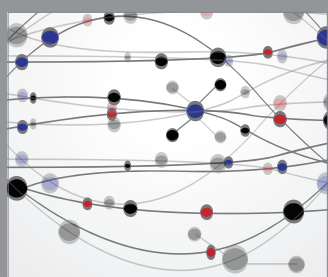

The Scientific World Journal

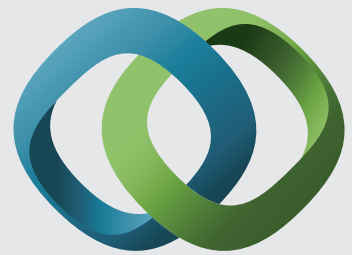

\section{Hindawi}

Submit your manuscripts at

https://www.hindawi.com
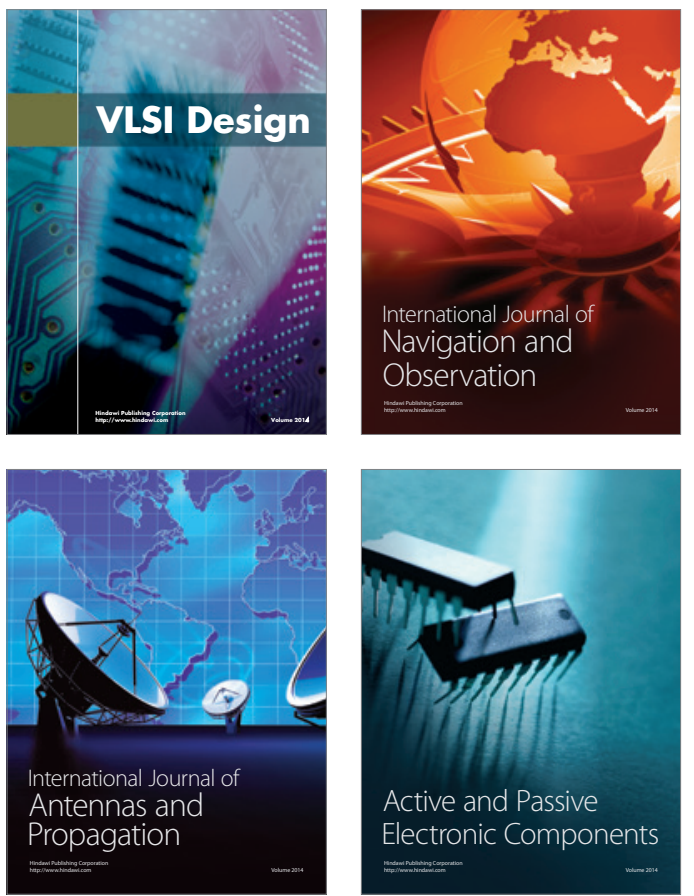
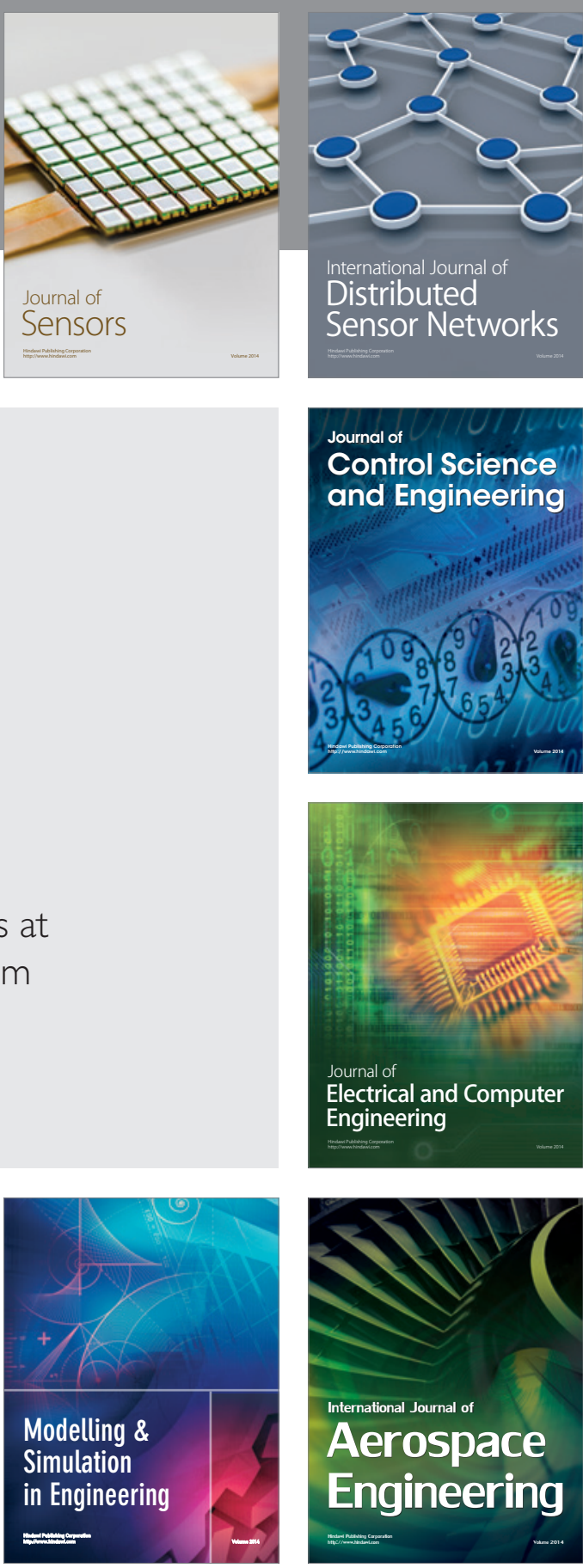

International Journal of

Distributed

Sensor Networks

$-$

Joumal of

Control Science

and Engineering
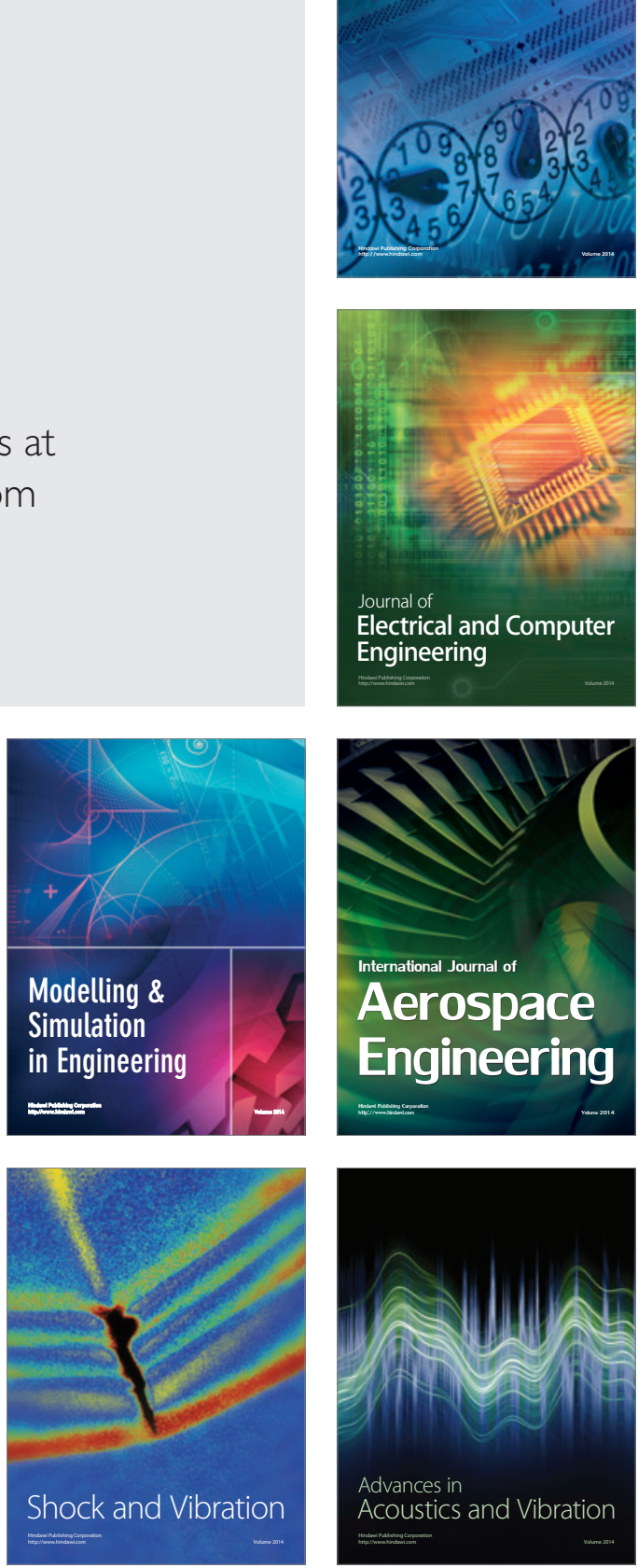